\title{
水化介孔氧化硅表面酸碱平衡常数的测定
}

\author{
张卫民杨振东刘嘉孙中溪 \\ (济南大学化学化工学院, 济南 250022)
}

\begin{abstract}
摘要：采用自动电位酸碱滴定技术研究了水化介孔氧化硅的表面脱质子反应. 基于悬浮液的酸碱滴定数据, 用 Gran 图法计算得到了介孔氧化硅表面氢离子键位点浓度. 利用 FITEQL 4.0 软件, 采用双电层恒电容模型计算得 到了相应的表面酸碱平衡常数. 结果表明, 与无定形氧化硅不同, 介孔氧化硅表面存在对应于双齿表面硅差基 $\equiv \mathrm{Si}_{2} \mathrm{OH}$ 和单齿表面硅羟基 $\equiv \mathrm{SiOH}$ 的两个脱质子常数, 分别为 $\mathrm{p} K_{\mathrm{al}}=6.78 \pm 0.15, \mathrm{p} K_{\mathrm{a} 2}=10.25 \pm 0.22$. 根据测定得出 的介孔氧化硅表面脱质子常数, 构建了不同 $\mathrm{pH}$ 条件下, 介孔氧化硅表面优势组分分布图, 并讨论了表面电容预 赋值对氧化硅表面组分分布的影响.
\end{abstract}

关键词: 平衡常数; 介孔氧化硅; 表面; 酸碱反应; 恒电容模型 中图分类号：0641

\section{Determination of Acid-Base Equilibrium Constants on Aqueous Mesoporous Silica Surfaces}

\author{
ZHANG Wei-Min YANG Zhen-Dong LIU Jia SUN Zhong-Xi* \\ (School of Chemistry \& Chemical Engineering, University of Jinan, Jinan 250022, P. R. China)
}

\begin{abstract}
We used the potentiometric titration technique to study the deprotonation reactions at aqueous mesoporous silica surfaces. The concentration of surface proton binding sites was obtained by the Gran plot method using the acid-base titration data. The relevant equilibrium constants of the surfaces in terms of the constant capacitance model (CCM) were determined based on the experimental results using the FITEQL 4.0 program. The results indicate that the surface deprotonation behavior of the mesoporous silica suspensions is significantly different from that of amorphous silica. This behavior can be described by two surface reactions with surface bidentate and monodentate deprotonation constants of $\mathrm{p} K_{\mathrm{al}}=6.78 \pm 0.15$ and $\mathrm{p} K_{\mathrm{a} 2}=10.25 \pm 0.22$, respectively. Using these deprotonation constants, we established a surface speciation diagram for mesoporous silica in aqueous suspensions as a function of $\mathrm{pH}$ and discussed the effect of surface capacitance on surface speciation.
\end{abstract}

Key Words : Equilibrium constant; Mesoporous silica; Surface; Acid-base reaction; Constant capacitance model

由于在催化剂、催化剂载体和吸附剂方面巨大的 应用潜力, 由介孔氧化硅的制备和表征引发的介 孔材料研究近年来一直是材料化学研究的前沿热 点 ${ }^{[1-4}$. 介孔氧化硅一般由硅溶胶粒子在有机模板剂 存在的条件下通过自组装形成, 在这个过程中氧化 硅表面与模板剂发生吸附等物理化学作用. 因此研
究氧化硅表面的化学反应对了解介孔氧化硅的形成 机理有重要意义. 然而, 相对于大量的关于介孔氧化 硅的制备、表征研究来说, 表面基本化学性质研究较 少, 表面酸碱性质的研究更是鲜见报道. 硅基介孔分 子篮的基本结构单元是硅氧四面体, 其表面上氧原 子位和硅原子位的未饱和键会与水溶液中的氢离子

Received: March 10, 2010; Revised: April 16, 2010; Published on Web: May 18, 2010.

*Corresponding author. Email: sunzx@ujn.edu.cn; Tel: +86-531-82765426.

The project was supported by the National Natural Science Foundation of China (20677022).

国家自然科学基金(20677022)资助项目

C. Editorial office of Acta Physico-Chimica Sinica 
和氢氧根离子发生反应而呈现出表面酸碱性质. 此 外, 硅氧四面体也是土壤及粘土矿物、催化剂、吸附 剂等的基本组成单元, 因此其表面酸碱反应和表面 络合行为对其催化和吸附性能影响极大, 值得深人 研究.

介孔氧化硅具有极大的比表面, 以介孔氧化物 作为表面化学反应研究的对象, 可保证足够的表面 键位的浓度, 从而减小测定过程的实验误差. 不同于 土壤和实际矿物样品, 人工合成的介孔氧化硅还可 以保证样品的纯度, 得到的平衡常数更具普适性. 对 于无定型氧化硅、高岭石、伊利石等天然含硅氧四面 体矿物的表面酸碱反应平衡常数已有文献报道 ${ }^{[5-7]}$, 而关于介孔氧化硅表面酸碱反应平衡常数尚未见报 道. 本文通过介孔氧化硅的酸碱滴定, 借助于计算机 软件 FITQEQL4.0, 采用恒电容模式计算了介孔氧 化硅表面酸碱反应平衡常数.

\section{1 实验部分}

\section{1 试剂与仪器}

实验用化学试剂正硅酸乙酯(TEOS)、十六烷基 三甲基溴化铵 $(\mathrm{CTAB}) 、$ 无水乙醇 $(\mathrm{EtOH}) 、 25 \%$ 氨水 $\left(\mathrm{NH}_{3} \cdot \mathrm{H}_{2} \mathrm{O}\right)$ 均为分析纯. 用二次蒸馏水来配制溶液.

主要仪器设备有美国 Beckman Coulter 公司 Avanti J-30I 型离心机、美国 Quantachrome 公司Nova 2000e 型比表面和孔隙度分布仪、瑞士万通 $702 \mathrm{SM}$ Titrino 自动电位滴定仪.

\section{2 介孔氧化硅的制备与表征}

采用传统的正硅酸乙酯水解法制备介孔 $\mathrm{SiO}_{2}$. 反应物配比为 $50 \mathrm{~mL} \mathrm{H}_{2} \mathrm{O}: 70 \mathrm{~mL}$ EtOH: $16 \mathrm{~mL} \mathrm{NH}_{3}$. $\mathrm{H}_{2} \mathrm{O}: 4.6 \mathrm{~mL}$ TEOS: $2.2 \mathrm{~g}$ CTAB. $80{ }^{\circ} \mathrm{C}$ 水解反应 $24 \mathrm{~h}$, 离心分离、干燥、813 K 煅烧 $6 \mathrm{~h}$ 制得样品.

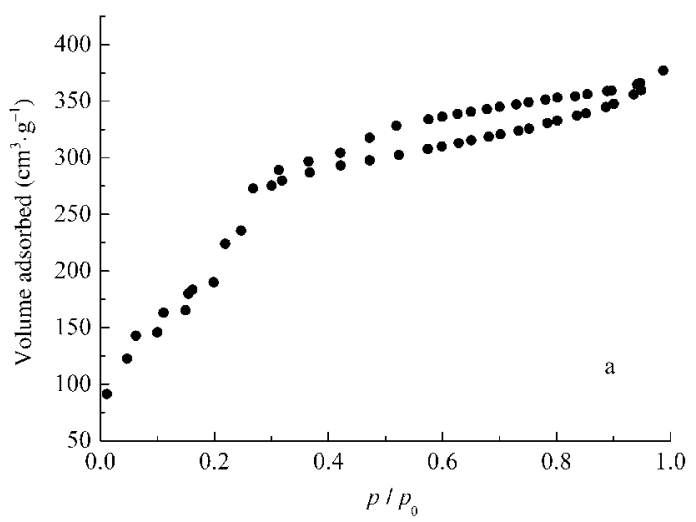

\section{3 酸碱滴定}

依次称取 $0 、 0.0202 、 0.0299 、 0.0404 \mathrm{~g}$ 氧化硅于 滴定槽中, 分别加人 $50 \mathrm{~mL} 0.1 \mathrm{~mol} \cdot \mathrm{L}^{-1} \mathrm{NaNO}_{3}$ 溶液. 选择等量滴定模式, 在高纯氮气保护的容器中和磁 力搅拌下, 预选择参数为摚拌 $2 \mathrm{~h}$, 步长 $0.1 \mathrm{~mL}$, 每 两滴间隔 $5 \mathrm{~min}$, 先用 $0.1008 \mathrm{~mol} \cdot \mathrm{L}^{-1} \mathrm{HCl}$ 溶液滴定 到 $\mathrm{pH}=3.0$, 停止酸量滴定; 再用 $0.1096 \mathrm{~mol} \cdot \mathrm{L}^{-1}$ 标准 $\mathrm{NaOH}$ 溶液反滴定到 $\mathrm{pH}=10.5$, 取反滴定数据计算 滴定过程中每一点酸的总浓度 $H_{\mathrm{t}}$, 作 $H_{\mathrm{t}}-\mathrm{pH}$ 图和 Gran 函数 $(G)$ 图, 计算介孔氧化硅表面羟基浓度.

\section{4 介孔氧化硅溶解度测定}

根据国标 GB602 配制硅标准溶液. 介孔氧化硅 悬浮液滴定结束后, 在 $20000 \mathrm{r} \cdot \mathrm{min}^{-1}$ 下离心 $30 \mathrm{~min}$, 取上清液采用硅钿蓝法 ${ }^{[8]}$ 测定游离的硅浓度 $C_{\mathrm{Si}}$.

\section{5 表面化学反应平衡常数的测定}

用计算机软件 FITEQ4.0 ${ }^{[9]}$ 计算氧化硅表面化学 反应平衡常数. 实验数据为每个滴定点的 $\mathrm{pH}$, 氢离 子总浓度和表面键位点浓度以及根据溶解度测出的 有关可溶性离子的总浓度. 为排除溶液中化学反应 对计算表面化学反应平衡常数的影响, 除可能的表 面化学反应和需优化计算的平衡常数外, 将体系中 所包含的有关溶液化学反应的平衡常数也依次输人 计算矩阵, 采用恒电容双电层模型来优化计算可能 存在的表面配位化学反应平衡常数. 可优化参数包 括表面化学反应平衡常数和双电层电容等. 计算机 计算结果的均方差 MSD 是实验数据和理论模型拟 合好坏的标志, MSD 值为 0.1-20 时结果较理想.

\section{2 结果与讨论}

\section{1 比表面和孔隙度测定}

$77 \mathrm{~K}$ 下 $\mathrm{N}_{2}$ 吸附/脱附等温线如图 1 所示, 在相

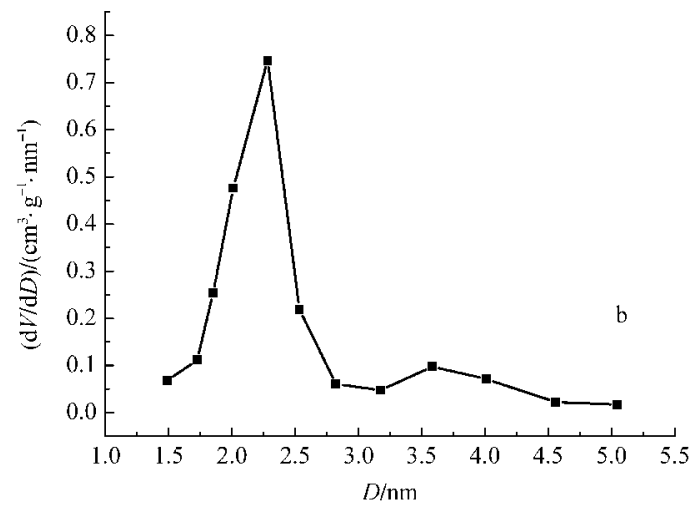

图 1 介孔 $\mathrm{SiO}_{2}$ 的 $\mathrm{N}_{2}$ 吸附/脱附等温线(a)和孔径分布图(b)

Fig.1 $\mathrm{N}_{2}$ adsorption/desorption isotherms (a) and pore size distribution (b) of mesoporous silica 
对压力 0.30-0.95 范围内出现明显的脱附滞后环. 低压范围内选择 6 个点计算样品的 BET 比表面积 为 $731.0 \mathrm{~m}^{2} \cdot \mathrm{g}^{-1}$, 以 $\mathrm{BJH}$ 模式计算脱附曲线得到最 可几孔径为 $2.28 \mathrm{~nm}$, 孔容为 $0.64 \mathrm{~cm}^{3} \cdot \mathrm{g}^{-1}$. 表明此法 得到了高比表面积、大孔容介孔 $\mathrm{SiO}_{2}$.

\section{2 氢离子在介孔氧化硅表面的吸附}

氢离子在介孔氧化硅表面的吸附可通过介孔氧 化硅悬浮液的酸碱滴定来检测. 当体系中溶解、沉淀 反应不起主导作用时, 溶液中游离氢离子的浓度受 表面化学反应制约. 氢离子在表面的吸附量可从添 加和游离的氢离子或氢氧根离子之差得到. 介孔氧 化硅悬浮液的酸碱滴定结果如图 2 所示.

$$
\begin{aligned}
& \text { 每一滴定点酸的总浓度 } \\
& H_{\mathrm{t}}=\left(V_{\mathrm{at}} \times C_{\mathrm{a}}-V_{\mathrm{b}} \times C_{\mathrm{b}}\right) /\left(V_{0}+V_{\mathrm{at}}+V_{\mathrm{b}}\right)
\end{aligned}
$$

其中 $C_{\mathrm{a}} 、 C_{\mathrm{b}}$ 分别为所滴加酸、碱的浓度, $V_{0} 、 V_{\mathrm{at}} 、 V_{\mathrm{b}}$ 分 别为初始体积、滴加酸、碱的体积. 反滴定至 $\mathrm{pH}>7$ 后, $H_{\mathrm{t}}$ 为负值. 由图 2 可见: 溶液在没有介孔氧化硅 存在时, 在 $\mathrm{pH}$ 值 6.5 到 10.0 范围内没有缓冲能力; 加人介孔氧化硅后呈现出明显的缓冲, 随着样品浓 度的增大缓冲能力逐渐增强; 在碱性范围内的缓冲 能力远大于酸性范围内的缓冲能力.

图 3 是介孔氧化硅悬浮液酸碱滴定过程中的 $G$ 函数与所加碱溶液体积的关系图. $G$ 函数与溶液中 游离氢离子浓度的关系如下:

$$
\begin{aligned}
& G=\left(V_{0}+V_{\mathrm{at}}+V_{\mathrm{b}}\right) \times 10^{-\mathrm{pH}} \quad \text { (acidic side) } \\
& G=\left(V_{0}+V_{\mathrm{at}}+V_{\mathrm{b}}\right) \times 10^{13.8 \mathrm{pH}} \quad \text { (alkaline side) }
\end{aligned}
$$

每一条散点图的左边是酸半支, 右边是碱半支, 酸半

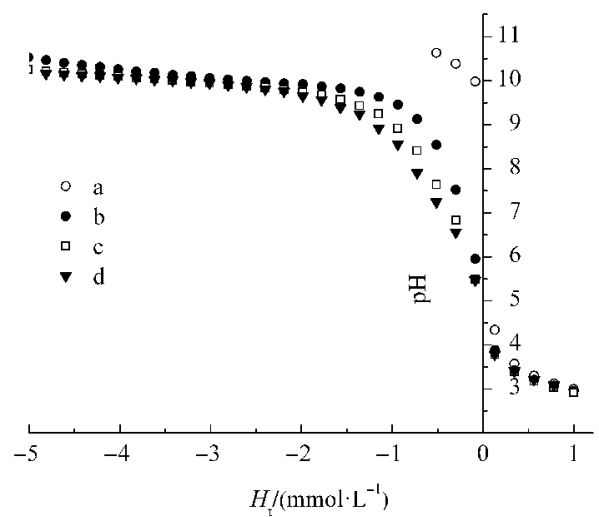

图 2 不同浓度介孔氧化硅 $\left(C_{\mathrm{s}}\right)$ 悬浮液的 $\mathrm{pH}$ 值与总酸浓度 的关系

Fig.2 pH of mesoporous silica suspensions with different concentrations $\left(C_{\mathrm{s}}\right)$ as a function of the concentration of the total $\mathrm{H}^{+}$ions added to the system $\left(\boldsymbol{H}_{\mathrm{t}}\right)$

$C_{\mathrm{s}} /\left(\mathrm{g} \cdot \mathrm{L}^{-1}\right)$ : (a) 0 , (b) 0.404, (c) 0.598, (d) 0.808

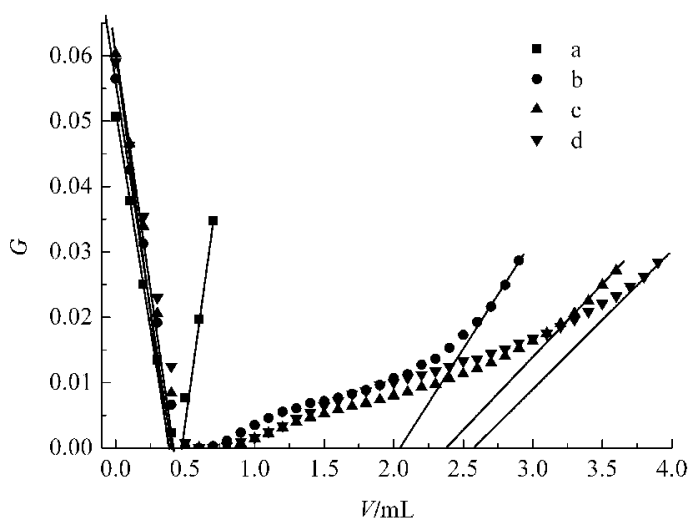

图 3 不同浓度介孔氧化硅固体悬浮液的 Gran 函数 $(G)$ 图

Fig.3 Gran functions $(G)$ for mesoporous silica of different solid concentrations

(a) $C_{\mathrm{s}}=0 \mathrm{~g} \cdot \mathrm{L}^{-1}, V_{\mathrm{el}}=0.400 \mathrm{~mL}, V_{\mathrm{ee} 2}=0.465 \mathrm{~mL}$; (b) $C_{\mathrm{s}}=0.404 \mathrm{~g} \cdot \mathrm{L}^{-1}, V_{\mathrm{el}}=$ $0.450 \mathrm{~mL}, V_{\mathrm{e} 2}=2.080 \mathrm{~mL}$; (c) $C_{\mathrm{s}}=0.598 \mathrm{~g} \cdot \mathrm{L}^{-1}, V_{\mathrm{el}}=0.460 \mathrm{~mL}, V_{\mathrm{e} 2}=2.420$ $\mathrm{mL}$; (d) $C_{\mathrm{s}}=0.808 \mathrm{~g} \cdot \mathrm{L}^{-1}, V_{\mathrm{el}}=0.470 \mathrm{~mL}, V_{\mathrm{e} 2}=2.600 \mathrm{~mL}$ $V_{\mathrm{el}}$ : volume of $\mathrm{NaOH}$ solution at equivalent point $\mathrm{l}$, $V_{\mathrm{e} 2}:$ volume of $\mathrm{NaOH}$ solution at equivalent point 2

支与碱半支分别线性回归与 $x$ 轴交点记为 $V_{\mathrm{el}}, V_{\mathrm{e} 2}$, 该方法基于在滴定过程中, 滴人的碱溶液先与溶液 中游离的酸反应再与表面上的质子反应, 当表面质 子反应完全以后, 溶液中的 $\mathrm{OH}^{-}$随碱的加人线性增 加, 因此, 用线形回归与 $x$ 轴的交点差值作为表面消 耗碱的体积, 该法是定量测定氢离子在表面上吸附 量的有效方法 ${ }^{[10-11]}$. 从图 3 可看出, 体系所消耗的氢 离子随固体含量的增加而增加. 根据 $G$ 函数图, 样 品表面氢离子键位点浓度 $H_{\mathrm{s}}$ 定义为

$$
H_{\mathrm{S}}=\left[\left(V_{\text {e2 sample }}-V_{\text {el sample }}\right) \times C_{\mathrm{b}}-\left(V_{\text {e2 blank }}-V_{\text {el blank }}\right) \times C_{\mathrm{b}}\right] / V_{\mathrm{b}}(4)
$$

因此, 可得出样品的单位表面吸附氢离子个数为

$$
D_{\mathrm{S}}=\left(H_{\mathrm{S}} \times N_{\mathrm{A}}\right) /\left(S \times C_{\mathrm{S}} \times 10^{18}\right)
$$

其中 $N_{\mathrm{A}}$ 为阿佛加德罗常数, $S$ 为 $\mathrm{N}_{2}$ 吸附/脱附 BET 比表面积, $C_{\mathrm{s}}$ 为固体总浓度.

以样品量为 $0 \mathrm{~g} \cdot \mathrm{L}^{-1}$ 作为空白, 对应 $V_{\mathrm{e} 1} 、 V_{\mathrm{e} 2}$ 为 $V_{\text {el blank }} V_{\mathrm{e} 2 \text { blank }}$; 则每一样品浓度均可获得一对应 $H_{\mathrm{S}}$ 、 $D_{\mathrm{s}}$. 不同固体浓度条件下, 悬浮液中 $H_{\mathrm{S}} 、 D_{\mathrm{S}}$ 和可溶性 硅的浓度 $C_{\mathrm{S}}$ 数据列于表 1.

\section{表 1 不同浓度介孔氧化硅悬浮液的表面位浓度 $\left(H_{\mathrm{S}}\right)$ 和} 表面位密度 $\left(D_{\mathrm{S}}\right)$

Table 1 Total surface site concentration $\left(H_{S}\right)$ and sarface site density $\left(D_{\mathrm{S}}\right)$ in silica suspension with different solid concentration

\begin{tabular}{cccc}
\hline$C_{\mathrm{s}} /\left(\mathrm{g} \cdot \mathrm{L}^{-1}\right)$ & $H_{\mathrm{s}} /\left(\mathrm{mmol} \cdot \mathrm{L}^{-1}\right)$ & $D_{\mathrm{s}} /\left(\mathrm{site} \cdot \mathrm{nm}^{-2}\right)$ & $C_{\mathrm{Si}} /\left(\mathrm{mmol} \cdot \mathrm{L}^{-1}\right)$ \\
\hline 0.404 & 3.397 & 6.9 & 1.225 \\
0.598 & 4.113 & 5.6 & 1.453 \\
0.808 & 4.482 & 4.2 & 1.857 \\
\hline
\end{tabular}

$C_{\mathrm{s}}$ : the concentration of silica dissolved in the system 
介孔氧化硅悬浮液非均相体系中, 随固体浓度 增加, 氢离子表面键位点浓度和可溶性硅浓度非线 性增加, 与颗粒间的絮凝、凝聚、离子的吸附、解吸等 过程同时存在有关, 反映了悬浮液体系化学反应的 复杂性. 据文献报道, 典型的氧化物表面每 $1 \mathrm{~nm}^{2}$ 含 有 4 至 10 个羟基 ${ }^{[12]}$, 实际数目取决于晶体结构的几 何形状、解理面本质、样品的预处理情况. 对于无定 形氧化硅单位表面氢离子个数, Schindler 等 ${ }^{[13]}$ 通过 酸碱反应法测定结果为 $5.83,5.46 \mathrm{~nm}^{-2}$, Davydov 等 ${ }^{[14]}$ 通过红外光谱和 $\mathrm{D}_{2} \mathrm{O}$ 交换法得到结果为 4.8 $\mathrm{nm}^{-2}$. 由于并非所有表面位均参与酸碱反应, 同时考 虑溶液离子强度、氧化物溶解的影响, 酸碱滴定法计

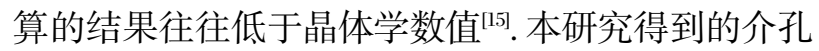
氧化硅单位表面羟基个数在 $4-7 \mathrm{~nm}^{-2}$ 之间, 数据合 理. 在后续计算表面酸碱平衡常数时, 我们发现氧化 硅表面羟基个数在 4-7 $\mathrm{nm}^{-2}$ 之间变化对平衡常数的 计算几乎没有影响, 从另一侧面证明了酸碱滴定法 测定 $D_{\mathrm{S}}$ 的合理性.

\section{3 介孔氧化硅的溶解}

计算介孔氧化硅的表面酸碱反应平衡常数时, 除考虑表面反应外, 还应考虑氧化硅的溶解. 与氧化 硅溶解有关的溶液化学反应及相关平衡常数如表 2 所示.

文献[16]报道, 无定形氧化硅悬浮液溶解生成 硅酸的量约为 $1-2 \mathrm{mmol} \cdot \mathrm{L}^{-1}$. 可溶性氧化硅的溶解 是一个缓慢的过程, 通常达到平衡需要几天 ${ }^{[17]}$, 且随 着 $\mathrm{pH}$ 的变化而变化. 为排除溶液化学反应对测定 固体表面化学反应平衡常数的影响, 本研究测定了 氧化硅溶解度最高时的游离浓度, 并将其输人计算 矩阵. 从表 2 和表 1 测定的游离硅组分浓度可以看 出, 游离硅的浓度远小于氢离子的表面键位点浓度, 并且可溶性硅组分的一级电离常数 $\mathrm{p} K_{\mathrm{a}}$ 值为 9.86, 说明在实验 $\mathrm{pH}$ 范围内, 溶液的 $\mathrm{pH}$ 缓冲作用主要源 自表面化学反应.
表 2 氧化硅悬浮液中的溶液化学反应 ${ }^{[18]}$

Table 2 Solution chemical reactions of soluble silicon species $^{[18]}$

\begin{tabular}{lr}
\hline Chemical reactions of silicic acid in aqueous solution & \multicolumn{1}{c}{$\mathrm{p} K$} \\
\hline $\mathrm{Si}(\mathrm{OH})_{4}=2 \mathrm{H}_{2} \mathrm{O}+\mathrm{SiO}_{2}$ & -2.71 \\
$\mathrm{Si}(\mathrm{OH})_{4}=\mathrm{H}^{+}+\mathrm{SiO}(\mathrm{OH})_{3}^{-}$ & 9.86 \\
$\mathrm{Si}(\mathrm{OH})_{4}=2 \mathrm{H}^{+}+\mathrm{SiO}_{2}(\mathrm{OH})_{2}^{2-}$ & 22.96 \\
$2 \mathrm{Si}(\mathrm{OH})_{4}=\mathrm{H}^{+}+\mathrm{Si}_{2} \mathrm{O}_{2}(\mathrm{OH})_{5}^{-}+\mathrm{H}_{2} \mathrm{O}$ & 8.10 \\
$2 \mathrm{Si}(\mathrm{OH})_{4}=2 \mathrm{H}^{+}+\mathrm{Si}_{2} \mathrm{O}_{3}(\mathrm{OH})_{4}^{2-}+\mathrm{H}_{2} \mathrm{O}$ & 19.76 \\
$3 \mathrm{Si}(\mathrm{OH})_{4}=3 \mathrm{H}^{+}+\mathrm{Si}_{3} \mathrm{O}_{6}(\mathrm{OH})_{3}^{3-}+3 \mathrm{H}_{2} \mathrm{O}$ & 28.60 \\
$3 \mathrm{Si}(\mathrm{OH})_{4}+\mathrm{H}_{2} \mathrm{O}=9 \mathrm{H}^{+}+\mathrm{Si}_{3} \mathrm{O}_{8}(\mathrm{OH})_{5}^{9-}$ & 27.50 \\
$4 \mathrm{Si}(\mathrm{OH})_{4}=3 \mathrm{H}^{+}+\mathrm{Si}_{4} \mathrm{O}_{7}(\mathrm{OH})_{5}^{3-}+4 \mathrm{H}_{2} \mathrm{O}$ & 25.50 \\
$4 \mathrm{Si}(\mathrm{OH})_{4}=4 \mathrm{H}^{+}+\mathrm{Si}_{4} \mathrm{O}_{8}(\mathrm{OH})_{4}^{4-}+4 \mathrm{H}_{2} \mathrm{O}$ & 35.94 \\
\hline
\end{tabular}

\section{4 介孔氧化硅的表面化学反应}

表面化学反应平衡常数与溶液化学反应平衡常 数的区别在于前者须考虑表面电荷的影响 ${ }^{[1]}$. 我们 通常所理解的平衡常数在表面化学中称作表观平衡 常数 $K_{(\mathrm{app})}^{\mathrm{s}}$, 它可以表示为表面固有常数 $K_{(\mathrm{int})}^{\mathrm{s}}$ 与静电 项的乘积:

$$
K_{\text {(app) }}^{\mathrm{s}}=K_{\text {(int) }}^{\mathrm{s}} \exp [-Z F \Psi /(R T)]
$$

式中 $Z$ 是表面电荷, $F$ 是法拉第常数, $\Psi$ 是表面电 位, $R$ 是摩尔气体常数, $T$ 是绝对温度. 在表面反应 过程中, 表面电荷是不断变化的, $K_{\text {(app) }}^{\mathrm{s}}$ 随之变化; $K_{\text {(int) }}^{\mathrm{s}}$ 是表面电荷为零时的平衡常数, 即所要计算的平衡 常数. $\Psi$ 与电容有关, 相应的表面络合模式有恒电容 模式、双层模式、三层模式、四层模式等. 本研究采用 恒电容模式 ${ }^{[19]}$ 来计算表面化学反应平衡常数.

对于表面酸碱反应模型, 通常也是最典型的模 型是设定均一表面, 存在两个表面化学反应, 一个脱 质子反应, 一个吸质子反应. 由于氧化硅带一个正电 荷的表面 $\equiv \mathrm{SiOH}_{2}^{+}$是强酸性组分, 只在强酸溶液中 存在 ${ }^{[20]}$, 所以在氧化硅表面只考虑脱质子反应, 如式 (7)所示.

$$
\equiv \mathrm{SiOH}=\equiv \mathrm{SiO}^{-}+\mathrm{H}^{+}
$$

借鉴无定形氧化硅的表面化学反应, 其表面脱 质子常数 $\mathrm{p} K_{\mathrm{a}}$ 为 $6.8 \pm 0.2^{[5]}$. 假定介孔氧化硅表面为

表 3 氧化硅悬浮液单表面模型表面参数

\begin{tabular}{|c|c|c|c|c|c|}
\hline$C_{\mathrm{s}} /\left(\mathrm{g} \cdot \mathrm{L}^{-1}\right)$ & $H_{\mathrm{S}} /\left(\mathrm{mmol} \cdot \mathrm{L}^{-1}\right)$ & $C_{\mathrm{Si}} /\left(\mathrm{mmol} \cdot \mathrm{L}^{-1}\right)$ & $C /\left(\mathrm{F} \cdot \mathrm{m}^{-2}\right)$ & $\mathrm{p} K_{\mathrm{al}}$ & MSD \\
\hline \multicolumn{6}{|c|}{$H_{\mathrm{S}}\left(D_{\mathrm{S}}=6.9\right)$} \\
\hline 0.404 & 3.397 & 1.225 & 100 & $9.97 \pm 0.02$ & 455.45 \\
\hline 0.598 & 5.028 & 1.453 & 100 & $10.07 \pm 0.02$ & 801.53 \\
\hline 0.808 & 6.794 & 1.857 & 100 & $10.15 \pm 0.02$ & 1014.00 \\
\hline \multicolumn{6}{|c|}{$H_{\mathrm{S}}$ (optimized by FITEQL) } \\
\hline 0.404 & 0.216 & 1.225 & 5 & $7.06 \pm 0.09$ & 7.80 \\
\hline 0.598 & 0.313 & 1.453 & 5 & $6.88 \pm 0.07$ & 7.08 \\
\hline 0.808 & 0.378 & 1.857 & 5 & $6.96 \pm 0.06$ & 11.15 \\
\hline
\end{tabular}

Table 3 Surface parameters of silica suspension using single surface chemical reaction model 
表 4 氧化硅悬浮液双表面模型的表面参数

Table 4 Surface parameters of silica suspension using two surface chemical reactions model

\begin{tabular}{|c|c|c|c|c|c|c|c|}
\hline$C_{\mathrm{s}} /\left(\mathrm{g} \cdot \mathrm{L}^{-1}\right)$ & $H_{\mathrm{Sl}} /\left(\mathrm{mmol} \cdot \mathrm{L}^{-1}\right)$ & $H_{\mathrm{S} 2} /\left(\mathrm{mmol} \cdot \mathrm{L}^{-1}\right)$ & $C_{\mathrm{Si}} /\left(\mathrm{mmol} \cdot \mathrm{L}^{-1}\right)$ & $C /\left(\mathrm{F} \cdot \mathrm{m}^{-2}\right)$ & $\mathrm{p} K_{\mathrm{al}}$ & $\mathrm{p} K_{\mathrm{a} 2}$ & MSD \\
\hline & \multicolumn{7}{|c|}{$H_{\mathrm{s}}\left(H_{\mathrm{s} 1}+H_{\mathrm{s} 2}\right)\left(\right.$ derived from $\left.D_{\mathrm{s}}=6.9\right)$} \\
\hline 0.404 & 0.356 & 3.040 & 1.225 & 100 & $6.68 \pm 0.016$ & $10.20 \pm 0.02$ & 18.55 \\
\hline \multirow[t]{2}{*}{0.808} & 0.712 & 6.081 & 1.857 & 100 & $6.88 \pm 0.010$ & $10.47 \pm 0.02$ & 12.67 \\
\hline & \multicolumn{7}{|c|}{$H_{\mathrm{S}}\left(H_{\mathrm{S} 1}+H_{\mathrm{S} 2}\right)\left(\right.$ derived from $\left.D_{\mathrm{S}}=5.6\right)$} \\
\hline \multirow[t]{2}{*}{0.808} & 0.712 & 4.845 & 1.857 & 100 & $6.89 \pm 0.010$ & $10.37 \pm 0.02$ & 13.92 \\
\hline & \multicolumn{7}{|c|}{$H_{\mathrm{S}}\left(H_{\mathrm{S} 1}+H_{\mathrm{S} 2}\right)\left(\right.$ derived from $\left.D_{\mathrm{S}}=4.2\right)$} \\
\hline 0.404 & 0.353 & 1.887 & 1.225 & 100 & $6.66 \pm 0.019$ & $10.14 \pm 0.02$ & 19.70 \\
\hline 0.598 & 0.524 & 2.793 & 1.453 & 100 & $6.92 \pm 0.013$ & $10.03 \pm 0.02$ & 19.00 \\
\hline
\end{tabular}

均一的表面硅差基 $\equiv \mathrm{SiOH}$, 发生脱质子反应形成带 负电的表面组分 $\equiv \mathrm{SiO}^{-}$. 按照脱质子表面化学反应 模型, 优化得到的参数示于表 3 . 从表 3 可以看出, 当不优化表面键位点浓度时, MSD 不能收玫在 20 以内, 而且 $\mathrm{p} K_{\mathrm{a}}$ 为 10.0 左右, 与文献报道值 6.8 有很 大差别. 表明介孔氧化硅具有不同于无定形氧化硅 的表面化学反应模型. 如果计算时优化表面浓度 $H_{\mathrm{S}}$, MSD 虽然可以收玫在 20 以内, 但是优化得出 $H_{\mathrm{S}}$ 值 仅为 $0.2-0.4 \mathrm{mmol} \cdot \mathrm{L}^{-1}$, 不足文献数值的 $10 \%$, 显然 不合理.

我们认为, 单表面模型不适合介孔氧化硅的表 面化学反应, 因此采用双表面模型优化计算. 双表面 模型将表面羟基分为单齿和双齿键合两种类型, 两 种键合点的酸碱性质不同. 表面 1 为两个硅原子与 一个羟基相连的双齿键合, 而表面 2 是一个硅原子 与一个羟基相连. 表面总浓度 $H_{\mathrm{S}}$ 根据 $D_{\mathrm{S}}$ 值分别等 于 6.9、5.6、4.2 计算得出, 每一种表面的具体浓度和

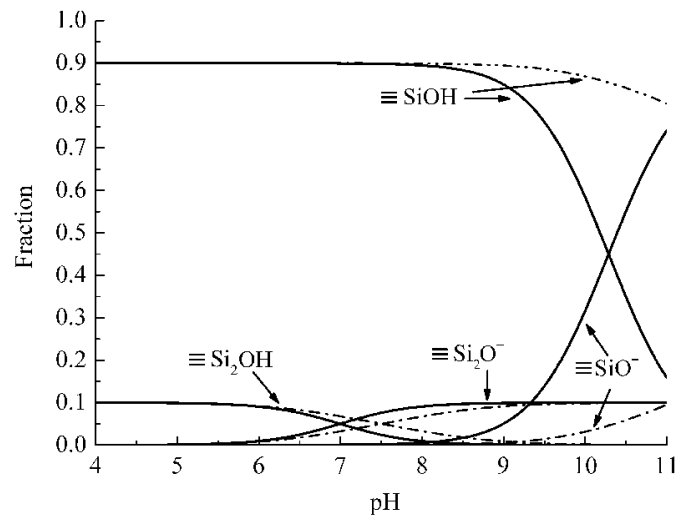

图 4 介孔氧化硅表面组分与 $\mathrm{pH}$ 的关系

Fig.4 Surface speciation of mesoporous silica as a function of $\mathbf{p H}$

The solid line represents the surface capacitance of $100 \mathrm{~F} \cdot \mathrm{m}^{-2}$ and the dash line represents the surface capacitance of $2 \mathrm{~F} \cdot \mathrm{m}^{-2}$.
比例由计算程序进行优化计算, 结果列于表 4. 得到 的 $\mathrm{p} K_{\mathrm{a} 1 、} \mathrm{p} K_{\mathrm{a} 2}$ 的变化范围分别为 6.63-6.92、10.0310.47. 即固体浓度和 $D_{\mathrm{S}}$ 取值不同, 对表面酸碱反应 平衡常数的影响并不显著.

$$
\text { 表面 } 1: \equiv \mathrm{Si}_{2} \mathrm{OH}=\equiv \mathrm{Si}_{2} \mathrm{O}^{-}+\mathrm{H}^{+}
$$

表面 $2: \equiv \mathrm{SiOH}=\equiv \mathrm{SiO}^{-}+\mathrm{H}^{+}$

表面酸碱反应平衡常数优化结果分别为: $\mathrm{p} K_{\mathrm{al}}=$ $6.78 \pm 0.15, \mathrm{p} K_{\mathrm{a} 2}=10.25 \pm 0.22$, MSD 收玫在 20 以内; 两个表面的浓度均与固体浓度成正比, $H_{\mathrm{S} 2}$ 比 $H_{\mathrm{S} 1}$ 大 一个数量级, 与滴定曲线一致, 即在 $\mathrm{pH}$ 为 10.0 时的 缓冲能力远远大于在 $\mathrm{pH}$ 为 6.8 时的缓冲能力. 意味 着相对于单齿键合表面硅羟基来说, 双齿键合表面 硅羟基的酸性更强, 符合多聚含氧酸酸性增强这一 基本原理. 另外, 硅酸溶液中的化学反应平衡常数表 明(表 2), 生成双核 $\mathrm{Si}_{2} \mathrm{O}_{2}(\mathrm{OH})_{5}^{-}$溶液组分脱质子反应 的 $\mathrm{p} K$ 值(8.10)小于生成单核 $\mathrm{SiO}(\mathrm{OH})_{3}^{-}$组分的 $\mathrm{p} K$ 值 (9.86), 也间接证明了本研究建立的介孔氧化硅双表 面模型的合理性.

\section{5 介孔氧化硅的表面组分分布}

根据优化得出的表面酸碱反应常数, 构建了介 孔氧化硅的表面组分分布与溶液 $\mathrm{pH}$ 的关系, 如图 4所示. 当表面电容为 $100 \mathrm{~F} \cdot \mathrm{m}^{-2}$ 、溶液 $\mathrm{pH}$ 小于 7.0 时, 两种表面键位的表面优势组分分别为 $\equiv \mathrm{SiOH}$ 和 $\equiv \mathrm{Si}_{2} \mathrm{OH}$; 而当溶液 $\mathrm{pH}$ 介于 7.0-10.2 之间, 表面 优势组分分别为 $\equiv \mathrm{SiOH}$ 和 $\equiv \mathrm{Si}_{2} \mathrm{O}^{-} ; \mathrm{pH}$ 大于 10.2 时, 为 $\equiv \mathrm{SiO}^{-}$和 $\equiv \mathrm{Si}_{2} \mathrm{O}^{-}$. 表面电荷对表面组分分布具 有重要影响, 表面电容减小为 $2 \mathrm{~F} \cdot \mathrm{m}^{-2}$ 时, 溶液优势 组分区域向右移动, 单齿键合表面硅羟基 $\equiv \mathrm{SiOH}$ 在 $\mathrm{pH}$ 小于 11 时始终占优势, 而 $\equiv \mathrm{Si}_{2} \mathrm{O}^{-}$的开始优势区 域由 $\mathrm{pH} 7.0$ 右移至 $\mathrm{pH}$ 7.5. 


\section{3 结 论}

以 $\mathrm{CTAB}$ 为模板、采用正硅酸乙酯水解法制备 了高比表面、大孔容介孔 $\mathrm{SiO}_{2}$. 与无定形氧化硅不同, 介孔 $\mathrm{SiO}_{2}$ 表面存在对应于双齿表面硅羟基 $\equiv \mathrm{Si}_{2} \mathrm{OH}$ 和单齿表面硅差基 $\equiv \mathrm{SiOH}$ 的两个脱质子常数, 分别 为 $\mathrm{p} K_{\mathrm{a} 1}=6.78 \pm 0.15, \mathrm{p} K_{\mathrm{a} 2}=10.25 \pm 0.22$.

\section{References}

1 Kresge, C. T.; Leonowicz, M. E.; Roth, W. J.; Vartuli, J. C.; Beck, J. S. Nature, 1992, 359: 710

2 Zhang, D. Q.; Wan, Y.; Li, H. X. Acta Chim. Sin., 2006, 64: 894 [张蝶青, 万 颖, 李和兴. 化学学报, 2006, 64: 894]

3 Li, J. S.; Gu, J.; Xia, M. Y.; Yuan, J. F.; Sun, X. Y.; Wang, L. J. Acta Chim. Sin., 2008, 66: 2305 [李健生, 顾 娟, 夏敏亚, 袁金芳, 孙秀云, 王连军. 化学学报, 2008, 66: 2305]

4 Zhang, H.; Wu, J.; Zhou, L.; Zhang, D.; Qi, L. Langmuir, 2007, 23: 1107

5 Schindler, P. W.; Kamber, H. R. Helv. Chim. Acta, 1968, 51: 1781

6 Du, Q.; Sun, Z.; Willis, F.; Tang, H. X. J. Colloid Interface Sci., 1997, 187: 221

7 Wang, Y.; Du, B.; Dou, X.; Liu, J; Shi, B.; Wang, D.; Tang, H.
Colloids and Surface A, 2007, 307: 16

8 Shen, Z.; Zhou, S.; Pei, S. Estuarine, Coastal and Shelf Science, 2008, 78: 481

9 Herbelin, A.; Westall, J. C. FITEQL ver 4.0. Corvallis, OR: Department of Chemistry, Oregon State, 1999

10 Pan, J.; Liu, R.; Tang, H. J. Environmental Sci., 2007, 19: 403

11 Sun, Z. X.; Guo, S. Y. Chem. J. Chin. Univ., 2006, 27: 1351 [孙中溪, 郭淑云. 高等学校化学学报, 2006, 27: 1351]

12 Huang, C.; Stumm, W. J. Colloid Interface Sci., 1973, 43: 409

13 Schindler, P. W.; Fürst, B.; Dick, R.; Wolf, P. U. J. Colloid Interface Sci., 1976, 55: 469

14 Davydov, V. Y.; Kiselev, A. V.; Zhuravlev, L. T. Trans. Faraday Soc., 1964, 60: 2254

15 Lützenkirchen, J.; Boily, J. F.; Lövgren, L.; Sjöberg, S. Geochimica et Cosmochimica Acta, 2002, 66: 3389

16 Charlet, I.; Schindler, P. W.; Spadini, L.; Furrer, G.; Zysset, M. Aquatic Science, 1993, 55: 291

17 Jordan, N.; Marmier, N.; Lomenech, C.; Giffaut, E.; Ehrhardt, J. J. J. Colloid Interface Sci., 2007, 312: 224

18 Puigdomenech, I. MEDUSA ver 2.0. Stockholm, Sweden: Royal Institute of Technology, 1999

19 Lützenkirchen, J. J. Colloid Interface Sci., 1999, 210: 384

20 Lagerstrom, G. Acta Chem. Scand., 1959, 13: 722 